\title{
Linear Discriminant Analysis for Classification of a Large Virtual Smart Meter Data Set With Known Building Parameters
}

\author{
Adam Neale, Michaël Kummert, Michel Bernier \\ Polytechnique Montréal, Montréal, Canada
}

\begin{abstract}
Linear discriminant analysis (LDA) classification is performed on a virtual smart meter (VSM) data set for 40000 buildings. LDA is used to classify the VSM data according to known building characteristics. The classification accuracy is evaluated based on the number of features and the number of smart meter data profiles used for classification. Some building parameters require a large number of data profiles to distinguish the class categories accurately. In most cases, the classification accuracy reached $90 \%$ or higher using 5 -fold crossvalidation. For example, the building location is well classified by LDA. However, some parameters such as building rotation and the building's aspect ratio are not properly discerned by the classification model. The results presented in this paper provide some insight into the effectiveness of LDA to accurately classify building parameters using smart meter data. The paper also describes a general methodology that can be used to apply LDA classification to smart meter data.
\end{abstract}

\section{Introduction}

Modeling of building stock energy consumption is a complex task that often requires simplified approaches. Building archetypes are one such method of bottom-up modeling that requires information to segment and characterize the chosen building stock. The accuracy of the archetypes depends largely on the quality and quantity of information available on the buildings. As a part of an ongoing study, a set of virtual electricity smart meter data with known building parameters was developed using batch building simulations (Neale et al. 2019). The intended use of the data set is to develop inverse models to extract information on the building from a set of anonymous electricity smart meter data. The VSM data set statistically represents a market of over 1 million single-family homes in the province of Québec (Canada).

\section{Smart meters and classification}

Smart meters for electricity consumption monitoring have become prevalent worldwide, with global investments on the order of 8.5 billion USD in 2015 (Metering and Smart Energy International 2018). Hydro-Québec recently completed a smart meter campaign that saw the installation of over 3.7 million smart meters in the province of Québec (Hydro-Québec 2016). While they are mostly used for billing purposes, the smart meter data represent a largely untapped resource that can provide insight into building performance and energy use at a relatively fine time scale. In Québec alone, 130 billion data points are collected each year.

At present, there are limited available smart meter data sets with known building parameters. As an example, there is the Irish Social Science Data Archive (ISSDA) Commission for Energy Regulation (CER) data set of Irish residential dwellings with over 4000 homes with smart meter data (CER 2012). The CER study focused primarily on the behaviour of the occupants rather than registering detailed physical characteristics of the dwellings.

Some have used the CER data set to evaluate machine learning classification algorithms (Beckel et al. 2014; Carroll et al. 2018). LDA was used and compared to other classification techniques with varying degrees of success. Carroll et al. (2018) used other supervised machine learning techniques, such as neural networks, to evaluate the accuracy of predicting the CER data set building parameters. In terms of applicability to the present study, since the building surface area was the only relevant building parameter included in the CER data set, additional investigation into smart meter classification was necessary. In general, smart meter data sets lack in key building parameters and often do not contain any information about the buildings.

\section{Objective and methodology}

The primary objective of this study is to provide a detailed analysis of linear discriminant analysis (LDA) classification for a (virtual) electricity smart meter data set with known building characteristics. A virtual smart meter data set is used in order to tie key building parameters to the electricity consumption patterns of the building. The impact of the number of features used to develop the classification model and the size of the data set on classification model accuracy is studied.

LDA classification can be used to separate a data set into several predefined classes corresponding to specific parameter values. For example, it could be used to sort a data set with the energy performance of a large number of buildings into categories corresponding to the number of occupants in the building. LDA is applied here to a VSM data set consisting of 40000 buildings and 19 known building parameters for each building. The accuracy and computational speed of the classification at various time scales (monthly, daily, hourly, etc.) is evaluated. Results 
are validated using a set of 30 houses with smart meter data and known building properties.

\section{A note regarding privacy concerns}

The extraction of information about a building brings the concern for the privacy of the occupants and the ethical ramifications of deducing information about their lifestyle and living habits. These concerns are mitigated in the case of this study due to the detachment between the real building and the identity of the occupants. In no cases will a building's true address be identified and associated with specific occupants. The general focus of the authors is on improving the building stock segmentation and characterisation processes used for building archetype (typology) development (see e.g. Reinhart and Cerezo Davila 2016). Results are processed at the building stock level and not for individual buildings.

\section{Linear discriminant analysis}

Linear discriminant analysis (LDA) is a supervised machine learning data classification technique that is used to establish a decision boundary based on a previously determined set of data. The developed LDA model is then used to classify new data.

An example data set with approximately 150 residential buildings with two features (electricity consumption on January $15^{\text {th }}$ and July $15^{\text {th }}$ ) and two categories (small and large homes) is illustrated in Figure 1. The building size is the class of the data set, and the objective of applying LDA to this example would be to classify new buildings as either small or large buildings based on their summer and winter electricity consumption.

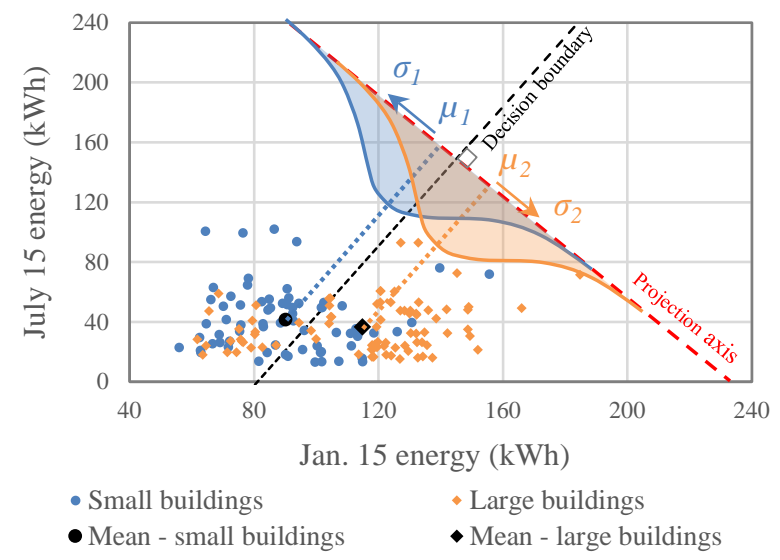

Figure 1: Linear classification example for a simple data set with two features and two categories.

The data illustrated in Figure 1 can be classified by projecting the data on a projection axis. The axis is determined by maximizing the difference between the projected means of the two data sets divided by the sum of the variance of each set, as described in Equation (1).

$$
\sim \operatorname{Max}\left[\frac{\left(\mu_{1}-\mu_{2}\right)^{2}}{\left(\sigma_{1}^{2}+\sigma_{2}^{2}\right)}\right]
$$

where $\mu$ is the mean for the data set and $\sigma^{2}$ is the variance. In more simple terms, the goal is to find the projection axis that will separate the two data groups as much as possible (maximizing the distance between the means) and clustering each individual set of data as much as possible (minimizing the variance). The result of maximizing the term in Equation (1) allows for the development of a linear equation of the form presented in Equation (2).

$$
Z=\beta_{1} X_{1}+\beta_{2} X_{2}
$$

where $Z$ is a cost constant, $\beta$ are coefficients calculated based on the covariance and mean values for the two data sets, and $X_{i}$ are the selected features of the set, in this case the energy consumption of the buildings for two specific days. $Z$ determines in part where the decision boundary crosses the projection axis. With multiple additional features, the equation takes the form illustrated in Equation (3).

$$
Z=\beta_{1} X_{1}+\beta_{2} X_{2}+\cdots+\beta_{j} X_{j}
$$

where $j$ is the number of features of the data set. With more than two features, the decision boundary is no longer linear and takes the form of a hyperplane. As the number of features increases, the system of equations to solve for the $\beta$ values is best expressed as a matrix equation, with the covariance matrix being of size $j \times j$.

It should be noted that the constant $Z$ is determined by the probability of a data point belonging to a specific category based on the amount of data in each category. This explains why the decision boundary does not lie mid way between the mean values of the two data sets illustrated in Figure 1. A large difference in the number of data points for one set will increase the probability of a new data point belonging to that set.

\section{Classification model accuracy}

The overlap of the two data sets in Figure 1 provides an understanding of the error involved in misclassification of new data points. Many points from each set lie on the socalled "wrong" side of the decision boundary. The accuracy of the model can be established based on dividing the data into training and validation sets. Holdover validation splits the data into two portions, reserving some percentage of the data for validation (such as $30 \%$ for validation, $70 \%$ for training). Cross-validation splits the data into a number of equal parts, also called folds. Each fold is used to validate the remaining portion of the data.

The main advantage of cross-validation over holdover validation is that it benefits from the entire data set for model training, but is memory and time intensive because it repeats the training and validation for each fold. This study uses 5-fold cross-validation for all of the classification model results. Classification model accuracy can be expressed using Equation (4).

$$
\text { Accuracy }=\frac{\text { Correct predictions }}{\text { Total predictions }}
$$

\section{Virtual smart meter data set}

As mentioned previously, one limitation of anonymous smart meter data is the lack of known building characteristics. Supervised machine learning is therefore normally not possible. As a part of a larger study, a set of virtual smart meter (VSM) data profiles were produced 
using a stochastic residential building stock model. The details of the larger study are described in (Neale et al. 2018). The VSM data set and stochastic model are described in detail in another publication (Neale et al. 2019).

In brief, the characteristics of each virtual building were generated stochastically based on probability distributions developed during segmentation and characterisation of the province of Québec's (Canada) single-family home (SFH) building stock. SFH are defined here as detached, semi-detached, and row houses. There are roughly 1 million SFH in Québec.

For the purpose of the present study, 40000 virtual buildings were produced by varying 19 stochastic building parameters, which include physical characteristics, such as the building envelope thermal performance values, and internal loads, such as the number of occupants and the appliance/lighting load profiles. The CREST thermal model was used to generate multiple stochastic occupancy and internal load profiles (McKenna and Thomson 2016). The CREST model can produce unique occupancy activity schedules with corresponding lighting, appliance and domestic hot water profiles using probability tables based on real appliance time-of-use data.

\section{Data aggregation}

Smart meter data are typically recorded at 15-minute intervals (see e.g. Hydro Québec 2012). On an annual basis this represents 35040 data points. With a large number of profiles the calculation time can become prohibitive. In order to simplify the exercise, the energy data can be aggregated to larger time scales. For the purpose of this study, the virtual smart meter profiles are aggregated on a monthly (12 data/profile), weekly (52 data/profile), daily (365 data/profile), and hourly (8760 data/profile) basis. Subsets of the annual data at 15minute intervals are also used, such as for a single month of the year, i.e. January (2976 data/profile).

\section{VSM classes}

As described previously, the classification process requires a data set to be described in terms of classes and features. The 19 stochastic building parameters described in Table 1, used to produce the VSM data profiles, were selected as the classes for the classification process. Categories describe the number of possible values for each class. Classes are divided into qualitative or quantitative variables. A qualitative variable has no numeric value, such as "electric heating” versus "nonelectric heating”. A quantitative variable would be a parameter with a numeric value, such as surface area.

The energy consumption values at each time step were used as the features of the data set. In doing so, the class categories were determined based on the variation in energy consumption between every time step. As an example, a building without air conditioning (AC) would have different mean and covariance values between winter and summer features than a building that does have AC, which the model can be trained to recognize.
Table 1: Building parameters used for classification (cat: categories, QL: qualitative, QN: quantitative)

\begin{tabular}{|c|c|c|c|}
\hline Class name & $\begin{array}{l}\# \\
\text { cat }\end{array}$ & Class description & Type \\
\hline Location & 7 & $\begin{array}{l}\text { Regions in the province of } \\
\text { Québec, e.g. Montreal, } \\
\text { Sherbrooke, etc. }\end{array}$ & QL \\
\hline $\begin{array}{l}\text { Building } \\
\text { type }\end{array}$ & 4 & $\begin{array}{l}\text { Type of residential building, } \\
\text { e.g. detached, semi-detached, } \\
\text { etc. }\end{array}$ & QL \\
\hline $\begin{array}{l}\text { Load } \\
\text { profile }\end{array}$ & 15 & $\begin{array}{l}\text { Stochastic occupancy and } \\
\text { internal load profiles }\end{array}$ & QN \\
\hline Window & 4 & Thermal performance levels & QL \\
\hline Area & 5 & $\begin{array}{l}\text { Heated building surface area } \\
\text { categorized by bin }\end{array}$ & QN \\
\hline WWR & 3 & Window-to-wall ratio & QN \\
\hline Rotation & 4 & $0,90,180,270$ degrees & QN \\
\hline Occupants & 5 & 1 to 5 occupants & QN \\
\hline $\begin{array}{l}\text { Adjacent } \\
\text { buildings }\end{array}$ & 4 & Detached, 1 shared wall, etc. & QL \\
\hline $\begin{array}{l}\text { Number of } \\
\text { floors }\end{array}$ & 2 & 1 or 2 floors & QN \\
\hline Wall RSI & 4 & $\begin{array}{l}\text { Vertical envelope thermal } \\
\text { performance values }\end{array}$ & QN \\
\hline Roof RSI & 6 & $\begin{array}{l}\text { Roof thermal performance } \\
\text { values }\end{array}$ & QN \\
\hline $\begin{array}{l}\text { Founda- } \\
\text { tion RSI }\end{array}$ & 4 & $\begin{array}{l}\text { Basement/foundation thermal } \\
\text { performance values }\end{array}$ & QN \\
\hline Leakage & 5 & Average air infiltration rates & QN \\
\hline $\begin{array}{l}\text { Air condi- } \\
\text { tioning }\end{array}$ & 3 & $\begin{array}{l}\text { No AC, window air } \\
\text { conditioner, heat pump. }\end{array}$ & QL \\
\hline Heat pump & 2 & $\begin{array}{l}\text { No heat pump, electric heat } \\
\text { pump }\end{array}$ & QL \\
\hline $\begin{array}{l}\text { Auxiliary } \\
\text { heating }\end{array}$ & 2 & $\begin{array}{l}\text { Electric or non-electric } \\
\text { heating }\end{array}$ & QL \\
\hline DHW type & 2 & Electric or non-electric DHW & QL \\
\hline $\begin{array}{l}\text { Aspect } \\
\text { ratio }\end{array}$ & 5 & $\begin{array}{l}\text { Building footprint aspect } \\
\text { ratio, e.g. } 0.8,1.2\end{array}$ & QN \\
\hline
\end{tabular}

\section{General classification approach}

The general procedure for developing a classification model can be described as follows:

1) Select the amount of buildings to be used for classification (up to 40000 in the case of this study), or $n$ profiles. This is the number of virtual smart meter profiles.

2) Select the time aggregation for the smart meter data (monthly, daily, etc.). This determines the number of features, $f$.

3) Select the class, $c l$, to be used to train the model (i.e. Location, building type, etc.). There are 19 class possibilities for this study, described in Table 1.

4) Train the model using linear discriminant analysis using the class values as the response for the model and the feature values as the predictors. 5-fold cross validation was used to determine the accuracy of the 
model. The response and predictor terminology is sometimes used in tools for classification.

5) Record the values for the classification model accuracy and modelling time for each scenario.

In order to evaluate the impact of the number of profiles and features on the classification accuracy, 760 classification model combinations were developed: 5 values of $n$ (1000, $5000,10000,20000,40000), 19$ possibilities of $\mathrm{cl}$ (19 parameters), and 8 values of $f$ ( 8 time aggregation scenarios).

\section{Results}

As described in the previous section, a large number of classification models were developed to investigate the impact of the number of features and profiles on the classification accuracy. The results are presented in terms of classification model accuracies, modelling time as a function of number of class categories, and some preliminary validation results with real smart meter data.

\section{Classification results}

The classification model accuracies (see Equation (4)) for $n=40000$ profiles for the 8 time aggregation scenarios and all 19 classes are illustrated in Table 2 . Similar tables exist for the other values of $n$ but are not presented in this paper for brevity. The results in Table 2 illustrate how the classification model accuracy varies for each class as a function of the number of features. The best result for each class is indicated by the bold line around the cell in the table. In the case of a tie, the scenario with the least number of features (and thus the fastest to compute) was chosen. The colour scale indicates the range of possible values, with red indicating a low result and green indicating a high result.

As a general rule, increasing the number of features has the effect of increasing the classification model accuracy, though as will be shown below this is not always the case and depends on the number of profiles used to classify the data.

Some classes are not well classified for the given data set using LDA. Building rotation and aspect ratio are essentially equal to the accuracy of randomly guessing the class category. For example, building rotation had 4 possible categories that were defined using a uniform probability distribution, and the resulting accuracy is equal to roughly 1 in 4 . This could be due to the relatively small impact that rotation has on the building energy and other model assumptions.

Other classes such as building location are well classified using LDA, even with a relatively low number of features. Since the location is tied to the weather file used for the building simulation, the impact on the energy consumption of the building is quite easily identified by the classification model, even at weekly and monthly data aggregation scales. Since the province of Québec has a heating-dominated climate, the classes that are directly tied to heating energy consumption are typically well classified. For example, whether a building has electric or non-electric auxiliary heating (class 17) is accurately captured here using LDA.

A smaller time period at a higher frequency, such as Scenario G, can provide good classification accuracy for classes that have a strong link to that period, i.e. heatingdominated effects for a heating-dominated period. Some

Table 2: Classification model accuracy for 40000 buildings and 19 classes. Values with bold borders are the best case for each class. WWR: window-to-wall ratio, RSI: thermal resistance in SI units, DHW: domestic hot water. Best viewed in colour where dark red $=0.0$ and dark green $=1.0$.

\begin{tabular}{|c|c|c|c|c|c|c|c|c|}
\hline \multirow{2}{*}{$\begin{array}{l}\text { Scenario: } \\
\text { Period: }\end{array}$} & A & B & $\mathbf{C}$ & D & $\mathbf{E}$ & $\mathbf{F}$ & $\mathbf{G}$ & $\mathbf{H}$ \\
\hline & 1 year & 1 year & 1 year & 1 year & Jan. 15 & July 15 & January & July \\
\hline Interval: & Monthly & Weekly & Daily & Hourly & 15-min & 15-min & 15-min & 15-min \\
\hline Features $(f)$ : & 12 & 52 & 365 & 8760 & 96 & 96 & 2976 & 2976 \\
\hline Classes $(c l)$ & \multicolumn{8}{|c|}{ Classification model accuracy } \\
\hline $1 \quad$ Location & 0.902 & 0.962 & 0.977 & 0.978 & 0.955 & 0.643 & 0.969 & 0.964 \\
\hline Building type & 0.800 & 0.800 & 0.834 & 0.932 & 0.794 & 0.800 & 0.932 & 0.796 \\
\hline Profile number & 0.283 & 0.826 & 1.000 & 1.000 & 0.961 & 0.986 & 1.000 & 1.000 \\
\hline Window & 0.497 & 0.537 & 0.692 & 0.921 & 0.475 & 0.406 & 0.914 & 0.532 \\
\hline Area & 0.437 & 0.454 & 0.590 & 0.859 & 0.434 & 0.383 & 0.892 & 0.472 \\
\hline WWR & 0.496 & 0.525 & 0.591 & 0.887 & 0.474 & 0.359 & 0.888 & 0.552 \\
\hline Rotation & 0.260 & 0.266 & 0.284 & 0.275 & 0.258 & 0.248 & 0.281 & 0.266 \\
\hline Occupants & 0.578 & 0.881 & 1.000 & 1.000 & 0.970 & 0.974 & 1.000 & 1.000 \\
\hline Adjacent buildings & 0.799 & 0.801 & 0.828 & 0.892 & 0.794 & 0.800 & 0.894 & 0.787 \\
\hline 10 Number of floors & 0.719 & 0.789 & 0.934 & 0.966 & 0.690 & 0.609 & 0.958 & 0.886 \\
\hline 11 Wall RSI & 0.598 & 0.633 & 0.719 & 0.889 & 0.599 & 0.591 & 0.893 & 0.654 \\
\hline Roof RSI & 0.308 & 0.352 & 0.511 & 0.784 & 0.297 & 0.209 & 0.786 & 0.364 \\
\hline 13 Foundation RSI & 0.558 & 0.604 & 0.807 & 0.881 & 0.498 & 0.486 & 0.883 & 0.755 \\
\hline $14 \quad$ Leakage & 0.419 & 0.493 & 0.715 & 0.680 & 0.430 & 0.205 & 0.796 & 0.526 \\
\hline Air conditioning & 0.804 & 0.815 & 0.888 & 0.914 & 0.784 & 0.833 & 0.799 & 0.901 \\
\hline $16 \quad$ Heat pump & 0.980 & 0.980 & 0.994 & 0.999 & 0.962 & 0.830 & 0.997 & 0.956 \\
\hline 17 Auxiliary heating & 0.996 & 0.997 & 1.000 & 1.000 & 0.986 & 0.959 & 1.000 & 0.981 \\
\hline 18 DHW type & 0.985 & 0.987 & 1.000 & 1.000 & 0.981 & 0.978 & 1.000 & 1.000 \\
\hline 19 Aspect ratio & 0.201 & 0.201 & 0.201 & 0.202 & 0.202 & 0.199 & 0.207 & 0.200 \\
\hline
\end{tabular}


potentially strange results can occur, such as achieving an $80 \%$ accuracy for air conditioning (AC) prediction using the month of January.

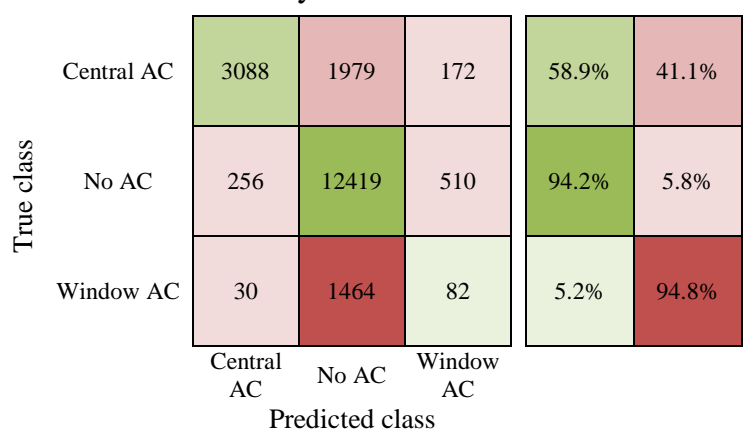

Figure 2: Confusion matrix for air conditioning classification using January smart meter data (Scenario G).

The confusion matrix in Figure 2 illustrates the number of true classes (rows) and predicted classes (columns) for the classification in matrix form. The diagonal matrix values contain the correctly predicted cases, i.e. the predicted class is the same as the true class. The two columns at the right of the matrix illustrate the percentage of correct and incorrect cases for each category in the class. As an example, there are 13185 houses without AC in the data set (66\% of the houses), of which 12419 have been assigned to the correct category, while 256 have been wrongly assigned to Central AC and 510 have been assigned to Window AC. This indicates that $94.2 \%$ of buildings without air conditioning were correctly identified.

As described previously, the classification model will tend towards the most probable outcome even if there is no link between the data (i.e. winter month energy consumption) and the class (i.e. is there air conditioning installed in the house). In the case of the considered building stock, 65\% of homes in Québec do not have air conditioning, which makes it likely to predict this outcome. A significant improvement in the prediction accuracy for air conditioning can be seen for Scenario $\mathrm{H}$ (July), illustrated in Figure 3.

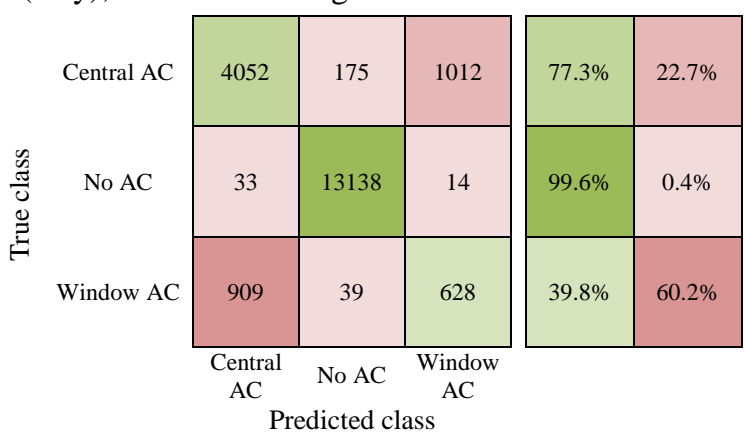

Figure 3: Confusion matrix for air conditioning classification using July smart meter data (Scenario H).

The confusion matrix in Figure 3 illustrates a net improvement for all categories of AC prediction. The main source of error is the confusion between the central $\mathrm{AC}$ and window AC categories, which is due to the relatively minor difference in coefficient of performance between the two cases. A logical improvement to this case could be to separate the class into only two categories: with and without air conditioning.

\section{Accuracy based on the number of features}

There is a direct link between the classification model accuracy and the number of features and profiles used to train the model. Figure 4 illustrates the accuracy results for the Location class as a function of the number of features and number of profiles.

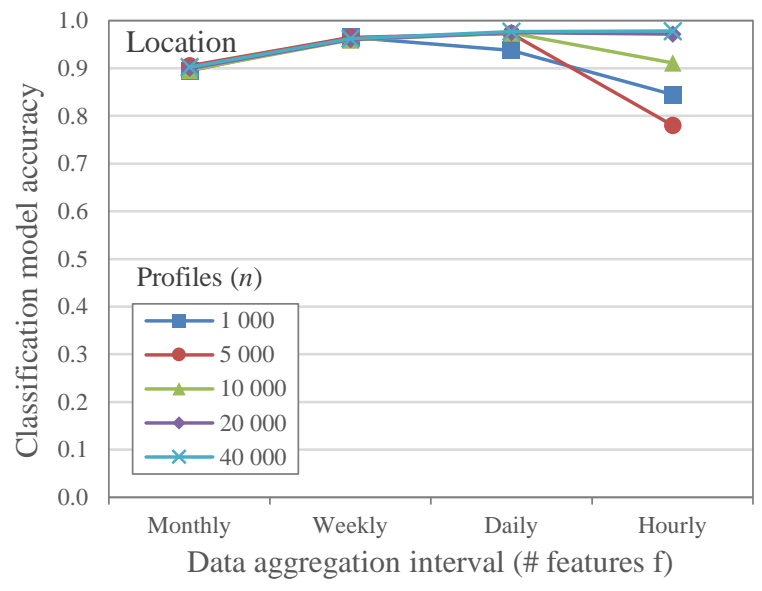

Figure 4: Location - classification model accuracy as a function of data aggregation interval.

The results in Figure 4 illustrate that there was little variation in classification model accuracy for monthly, weekly and daily time aggregation values, even for as few as 1000 buildings used to train the model. At an hourly time aggregation with 8760 features the accuracy begins to be impacted by the number of buildings. The noise level for hourly values of energy consumption requires a minimum number of buildings for accurate training. This effect is exacerbated for other classes, such as for building type illustrated in Figure 5.

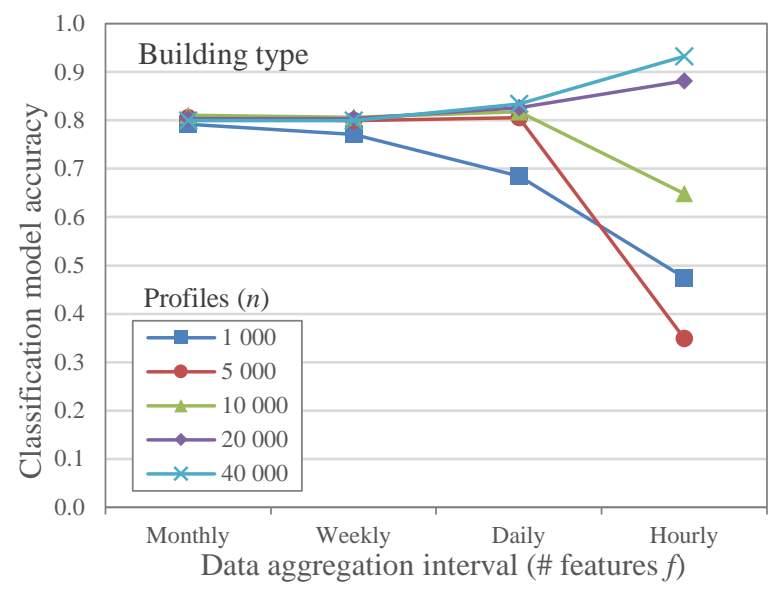

Figure 5: Building type - classification model accuracy.

There is an increased separation of classification model accuracies for hourly data illustrated in Figure 5, with values ranging from roughly 0.35 to over 0.90 . This is a large discrepancy in terms of model accuracy, which results from drawing incorrect conclusions due to the 
noise level on the energy consumption data at hourly values. At monthly values the accuracy of the classification process is higher, indicating it would be worse to attempt to classify on an hourly basis without a certain minimum sample of buildings for this case.

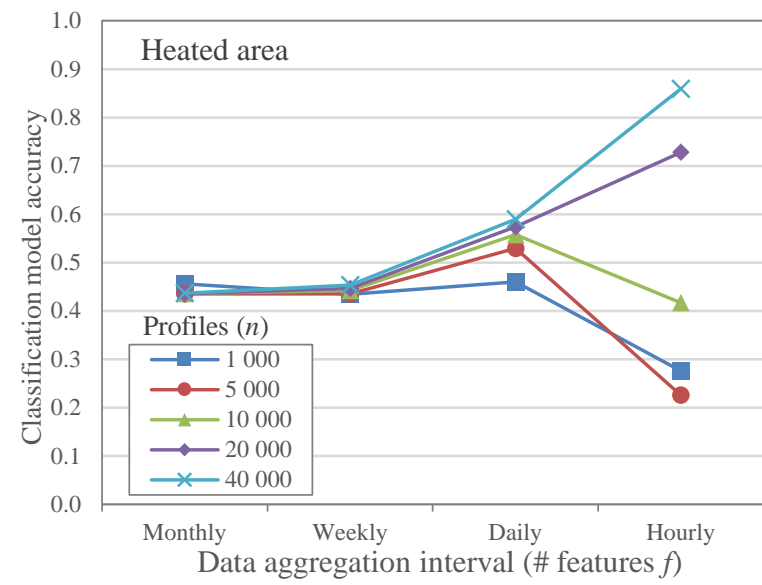

Figure 6: Heated area - classification model accuracy.

In the case of the heated area of the buildings, illustrated in Figure 6, the base level accuracy of the classification for monthly aggregation is much lower than for the building type (roughly 0.45 versus 0.80 accuracy). There is a significant improvement overall in increasing the number of features to daily or hourly levels, as long as there is a sufficient amount of building profiles to train the model.

Finally, in the previous cases there was a net improvement in classification accuracy for using hourly time aggregation, as long as a sufficient number of buildings profiles were used to capture the behaviour for each class. For some cases however, there was evidence that the classification accuracy could be further improved with additional building profiles for training.

Consider the classification model accuracy results illustrated in Figure 7 for the air infiltration rate of the building. The accuracy results have not converged in the same way as some of the other classes. There appears to be the potential for further improvement of the model
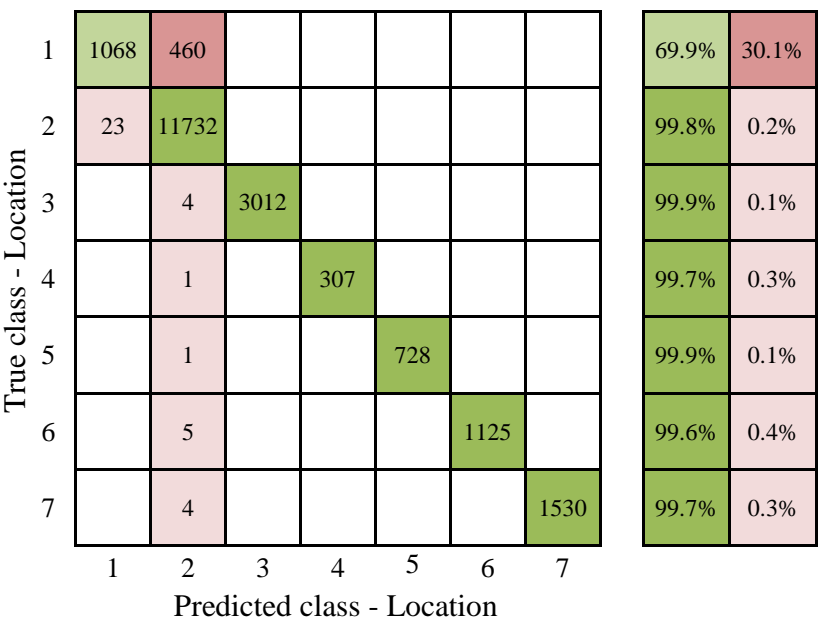

Figure 8. Confusion matrices for 20000 cases (left) and a subset of 17619 buildings with only electric auxiliary heating (right) based on the building location classes for 365 features (daily energy).

accuracy if the model were to be trained with additional building profiles.

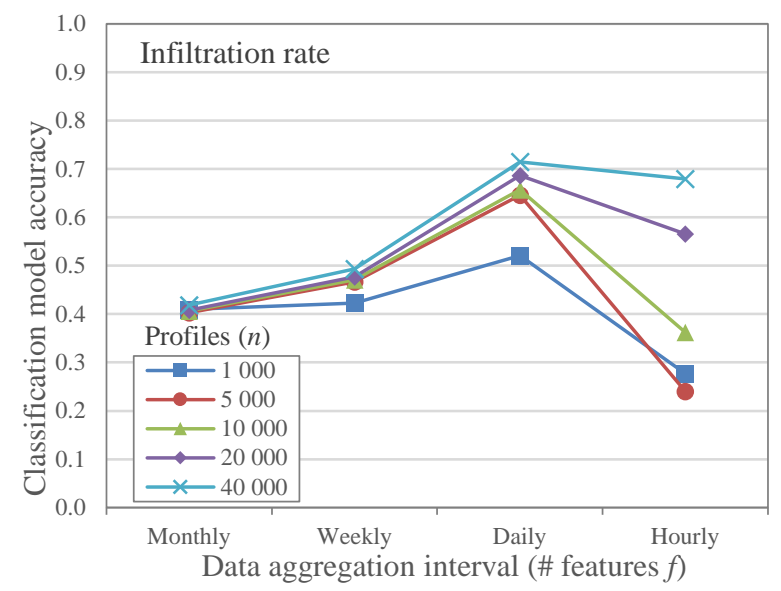

Figure 7: Infiltration rate - classification model accuracy.

\section{Improving classification accuracy}

In some cases, the classification model accuracies can be used to establish decision criteria for the classification approach that can be used to improve the results. The confusion matrix in Figure 8 (left) illustrates the true class categories and predicted class categories for the location of a set of 20000 virtual buildings. The accuracy for each category is indicated towards the right of the matrix. The overall classification model accuracy for this particular case is equal to $97.5 \%$. Upon closer review, this value may give a false impression of near perfect accuracy. The accuracy for the Location \#1 category for the location class is actually only $69.9 \%$, which indicates that while other categories are well classified, there is some confusion between the Location \#1 and Location \#2 results.

The two confused locations share a common trait in that they have a higher prevalence for non-electric auxiliary heating, such as natural gas or oil furnaces. Other regions in the province of Québec tend to have mainly electric heating due to the lower cost of hydroelectricity. With non-electric heating, the electricity load profiles for Location \#1 and \#2 become similar in magnitude, to the
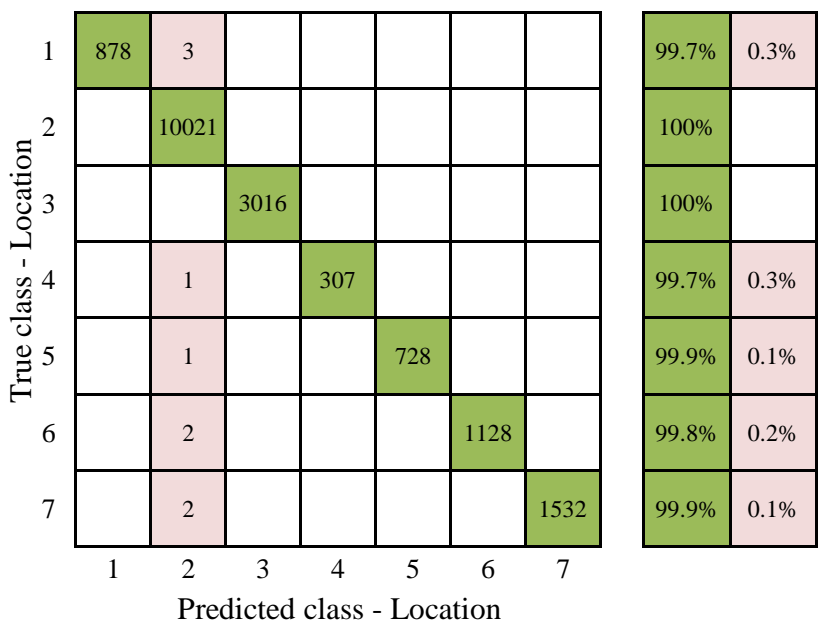
point where many buildings can not easily be distinguished by the classification model.

If the non-electric heating buildings are filtered out of the data set, leaving 17619 cases, the classification model achieves a near perfect accuracy and there is no longer any confusion in the classification model results as shown on the right portion of Figure 8.

In this case, the classification process can be improved by first determining the type of auxiliary heating and then by developing auxiliary-heating-dependant classification models based on the location of the building. While this requires a case-by-case understanding of the building stock characteristics and how they vary, it can be a useful means of improving classification results.

\section{Computational time}

The classification models were developed on an Intel i78700K@3.70 GHz computer with 64 GB of RAM with a solid-state hard drive. The classification model development time per number of categories for cases with 8760 features is illustrated in Figure 9.

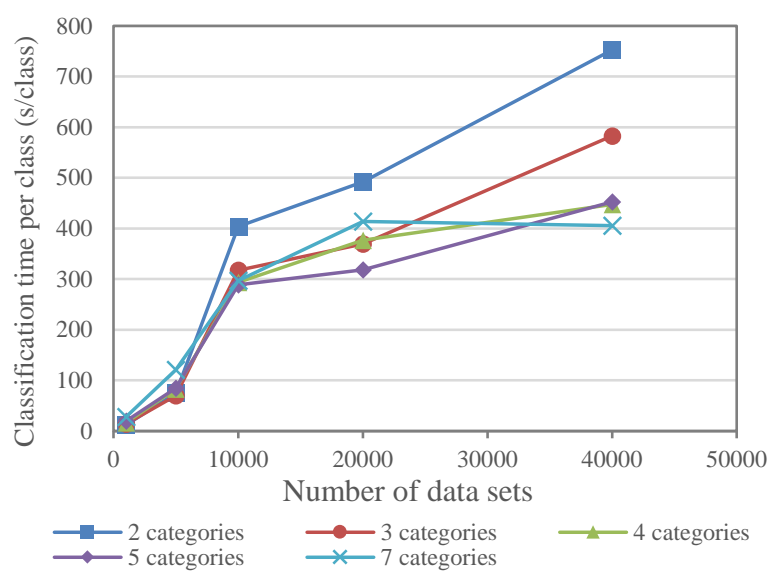

Figure 9: Computational time per class required to develop the classification models (8760 features).

There is a somewhat linear tendency when considering the classification time per class. The process seems to become more efficient as the number of categories increases. Some small variations in this trend are visible, in particular for the " 4 categories" and "7 categories" cases. One possible explanation is due to the nature of the classes that are represented. Since each curve represents a different class, it is possible that the difference in modelling times represent the complexity of developing a classification model for certain parameters.

\section{Validation}

The goal of the classification process is ultimately to be able to predict what the category would be for a given class for a set of real smart meter data. In the larger study of the authors, the resulting predicted class is used as an initial guess for a more elaborate calibration process, as a means of reducing the overall modelling time. Therefore, while it is desirable to predict the exact category for the real building, a value close to the desired result can also be acceptable. With this in mind, the predicted class categories can be described using the following terminology:

1) Hits - the category is correctly predicted;

2) Near - the category is closely predicted;

3) Misses - the category is incorrectly predicted.

The concept of near predictions depends on the class that is studied. For example, for building surface area, a near value could be one area bin higher or lower than the correct surface area bin. For location, near could be described as a predicted region with the same heating degree-days but not corresponding to the actual location of the building. For some classes there is no such concept of near values, such as the "electric" or "non-electric" categories of domestic hot water energy source.

As a first step towards validating the classification models, a set of 30 residential smart meter electricity consumption profiles for homes in one region of the province of Québec was used. Some characteristics of the homes were known and therefore were used to validate the predicted class categories.

The classification models developed for the location class were used to predict the region where the 30 homes were located, with exact matches corresponding to "hits" and regions with very similar heating degree-days corresponding to "near" results. The results are illustrated in Figure 10 for varying numbers of features (monthly to hourly time aggregation) and numbers of profiles (1 000 to 40000 profiles), for the location class category prediction accuracy.

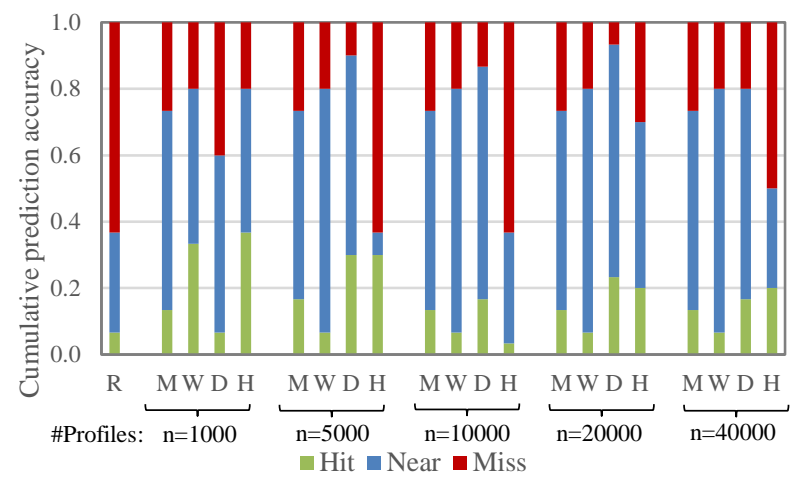

Figure 10: Location class category prediction results for a set of 30 houses for different training set sizes (n) and features.

$R$ : random guess, $M$ : monthly, W: weekly, D: daily, H: hourly.

The results in Figure 10 indicate a generally increasing trend in prediction accuracy based on the number of features, at least until daily time aggregation. For hourly results, the prediction accuracy seems variable. When considering the combined "hit" and "near" values, the prediction seems generally adequate as an initial guess of the regional conditions where the buildings are located, especially when compared with simply randomly guessing the location ("R" in Figure 10).

The data set used for validation has a number of issues that can explain the room for improvement in the prediction accuracy. First, 30 houses is a small sample when compared to the training set of up to 40000 buildings. Conditions could vary in specific ways that do 
not match the training set. Secondly, some aspects of building electricity use are not yet implemented in the virtual building model, i.e. swimming pool pumps and heaters. Finally, the real smart meter data was found to have a non-negligible amount of missing values. In some cases, this reached as high as $8 \%$ missing data, which had an unknown effect on the classification model accuracy. Further investigation into the prediction accuracy is required, which includes using a much larger real smart meter data set with a lower amount of missing data.

\section{Conclusion}

Classification can be a useful tool to interpret a data set and predict the class category for new data points. Using linear discriminant analysis (LDA) on a virtual smart meter data set with known building parameters can provide insight into the relationship between those characteristics and the energy consumption of a building.

LDA was successfully applied to 19 different building parameters, with 8 different feature scenarios and 5 data sets of different sizes. The classification model accuracy was determined using 5-fold cross-validation, which provided some insight into the effectiveness of LDA for the different classes examined.

Classification accuracy varied significantly based on the sample of data used to develop the classification model and as a function of the number of features. Some parameters, such as the building location, could be accurately predicted with a low number of features, such as with monthly or weekly aggregated data. With hourly energy data, a high sample of buildings was required to accurately capture the variance in the electricity consumption data. Due to the non-uniform probabilistic nature of some of the virtual building parameters, some results indicated a high accuracy when in reality the model was simply defaulting to the most likely outcome for the class categories. Caution is recommended for interpreting classification model accuracy for these types of cases. The confusion matrix can be a useful tool for interpreting the results and avoiding misinterpretation of the model effectiveness.

Classification models for hourly feature intervals required a large number of building profiles to reach the highest levels of accuracy. This is due to the highly stochastic nature of the electricity consumption at hourly intervals. In order to detect the correct class category, a large number of different cases were required to develop the model, to differentiate between the effects of the studied building parameter and other classes. There is clearly a trade-off between accuracy and the number of required features, where a classification modeller must choose between the complexity of the data set (i.e. $40000+$ profiles) and the desired model accuracy. Some building parameters also simply have less impact on electricity consumption, which explains the variability in classification accuracy.

Finally, the classification model accuracy was validated using a set of real smart meter data representing 30 residential homes in the province of Québec. The results indicate a reasonable prediction of the home's location, though some issues with the smart meter data will require further investigation. In particular, the effect of missing data on classification model prediction must be clearly established.

As the VSM data is a synthetic data set, the next step of this work will improve upon the stochastic residential building stock model used to generate the virtual smart meter profiles and investigate additional classification algorithms to see the impact on the classification model accuracy, with validation using additional real smart meter data. The goal will be to improve the virtual model to be as representative of the real building stock model as possible, to improve upon the classification and then test the accuracy of the classification models to predict multiple building parameters for real smart meter data.

\section{Acknowledgements}

The authors gratefully acknowledge the financial support received from an IVADO Fundamental Research Grant.

\section{References}

Beckel, Christian, Leyna Sadamori, Thorsten Staake, and Silvia Santini. 2014. "Revealing Household Characteristics from Smart Meter Data.” Energy 78: 397-410.

Carroll, Paula, Tadhg Murphy, Michael Hanley, Daniel Dempsey, and John Dunne. 2018. "Household Classification Using Smart Meter Data.” Journal of Official Statistics 34 (1): 1-25.

CER. 2012. CER Smart Metering Project - Electricity Customer Behaviour Trial, 2009-2010. 1st ed. Irish Social Science Data Archive. SN: 0012-00.

Hydro-Québec. 2012. Réponse d'Hydro-Québec Distribution Aux Engagements 3, 10, 15, 18, 21 (UC), 21 (UMQ) ET 22 À 27.

Hydro-Québec. 2016. Rapport Annuel 2015. Montréal, Canada.

McKenna, Eoghan, and Murray Thomson. 2016. "HighResolution Stochastic Integrated Thermalelectrical Domestic Demand Model." Applied Energy 165: 445-461.

Metering and Smart Energy International. 2018. "Global Trends in Smart Metering,” December.

Neale, Adam, Michaël Kummert, and Michel Bernier. 2018. "Generator: A Stochastic Virtual Smart Meter Data Generation Model for Residential Building Stock Characterization.” In ESim 2018, the 10th Conference of IBPSA-Canada, 65-74. Montreal, Canada.

Neale, Adam, Michaël Kummert, and Michel Bernier. 2019. "Developing a Stochastic Virtual Smart Meter Data Set for Residential Buildings Methodology and Sample Data.” In Preparation.

Reinhart, Christoph F., and Carlos Cerezo Davila. 2016. "Urban Building Energy Modeling - A Review of a Nascent Field.” Building and Environment 97: 196202. 